\title{
Friction Observer and Compensation for Control of Robots with Joint Torque Measurement
}

\author{
Luc Le Tien*, Alin Albu-Schäffer*, Alessandro De Luca**, and Gerd Hirzinger* \\ (**) Università di Roma "La Sapienza" \\ (*) German Aerospace Center (DLR) \\ Oberpfaffenhofen, D-82234 Weßling, Germany, http://www.dlr.de/rm/
}

\begin{abstract}
In this paper we introduce a friction observer for robots with joint torque sensing (in particular for the DLR medical robot) in order to increase the positioning accuracy and the performance of torque control. The observer output corresponds to the low-pass filtered friction torque. It is used for friction compensation in conjunction with a MIMO controller designed for flexible joint arms. A passivity analysis is done for this friction compensation, allowing a Lyapunov based convergence analysis in the context of the nonlinear robot dynamics. For the complete controlled system, global asymptotic stability can be shown. Experimental results validate the practical efficiency of the approach.
\end{abstract}

Index Terms - Friction compensation, friction observer, flexible joint robots

\section{INTRODUCTION}

For robots with high gear ratio aiming at low own weight and high payload, as the DLR medical robot (Fig. 1, [1]), the effects of joint friction can strongly influence the system performance. On the other hand, for medical applications such as minimal invasive surgery or bone cutting and milling for prosthetics, the accuracy of the system plays a central role, motivating the friction compensation development of this paper.

Friction compensation is a very basic problem in motion control and therefore there exists a huge amount of literature on this topic, out of which only a small fraction can be cited here. The approaches include model-based friction compensation [2], [3], adaptive controllers [4], [5], sliding mode control [6], classical integrator action and disturbance observers [7]. Since the parameters of friction strongly vary with temperature and with time, model-based friction compensation is quite inaccurate. Adaptive and sliding mode

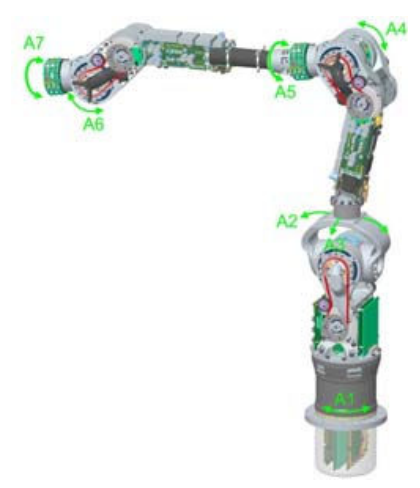

Fig. 1. The torque controlled DLR medical robot arm techniques adapt to parameter variations, are however sensitive to unmodelled dynamics. On the other hand, standard linear techniques such as integrators or disturbance observers are typically used in industrial robotics applications and show good practical performance. Their analysis, however, is usually based on linear techniques and does not really apply to the strongly nonlinear robotic systems. For integrators, only local convergence results have being achieved in robotics [8].

The approach proposed in this paper is based on a friction observer inspired by the momentum-based fault and collision detection algorithms developed in [9], [10]. The observer is shown to provide a low pass filtered disturbance torque, which is further used for friction compensation. Although for the linear case this observer can be shown to be equivalent to other disturbance observer techniques or to integrator based controllers, we are interested here in the development and analysis of an observer which works in conjunction with a passivity-based MIMO controller acting on the full nonlinear robotic system. The presented approach has the advantage to enable a passivity analysis, which allows in turn the treatment of the controller in a Lyapunov framework leading to global asymptotic results. Since in the DLR medical robot the joint torque is measured after the gearbox, one can distinguish between external loads acting on the link side of the robot and the internal friction disturbance acting mostly on the actuator. Hence, the same observer technique can be used to independently determine the two different disturbance torques. Detection/reaction to collision forces on the link side was presented in [10], while observation/compensation of friction on the motor side is the topic of the current paper. The paper is organized as follows. Section II introduces the main idea on the simple case of one actuator, but the presentation and analysis are done so that the results can be directly applied to the whole multi-dof robot. Section III introduces the robot model, while Sec. IV summarizes the controller and the convergence analysis results from [14], obtained for ideal friction compensation. Combining these results, Sec. V discusses the stability of the systems with the new friction compensation. Finally, the obtained performance is verified by experimental tests reported in Sec. VI.

\section{OBSERVER DESIGN IDEA AND PASSIVITy ANALYSIS}

Consider a robot with a passive controller as described in Fig. 2. The robot model can be further subdivided into the 
nonlinear robot dynamics ${ }^{1}$ and the passive actuator block,

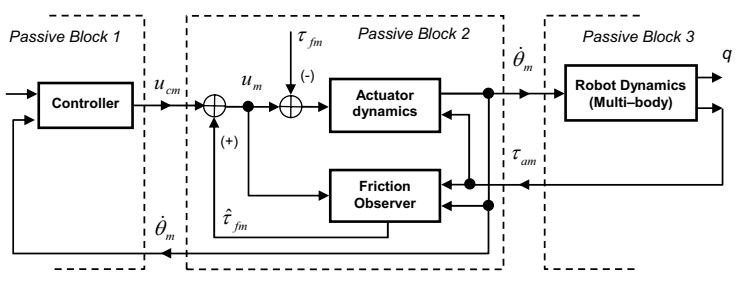

Fig. 2. Overview of the system as interconnection of passive blocks. For a more detailed representation of the passive structure of the plant see also Fig. 6.

which is linear except for the friction disturbance. In this section we will address only a single actuator in order to introduce the design idea. The actuator dynamics is described by

$$
u_{m}=J_{m} \ddot{\theta}_{m}+\tau_{a m}+\tau_{f m} .
$$

Therein, $u_{m}$ is the motor torque, $\theta_{m}$ is the motor position, $J_{m}$ is the motor inertia, and $\tau_{a m}$ and $\tau_{f m}$ are the joint torque (including visco-elasticity effects of the transmissions ${ }^{2}$ ) and the friction torque, respectively. Obviously the subsystem is passive, with the storage function

$$
S_{\theta}=\frac{1}{2} J_{m} \dot{\theta}_{m}^{2}
$$

and its derivative

$$
\dot{S}_{\theta}=\dot{\theta}_{m} u_{m}-\dot{\theta}_{m} \tau_{a m}-\dot{\theta}_{m} \tau_{f m} .
$$

On the right hand side, the first term is the power supplied by the controller, the second term is the power transmitted to the links. The last term is the power dissipated due to friction and is of course always negative semi-definite. In particular, we consider for the analysis in this paper the following standard friction model containing Coulomb friction $\tau_{f m, c}$ and viscous friction $\tau_{f m, v}$

$$
\tau_{f m}=\tau_{f m, c}+\tau_{f m, v}=f_{c} \operatorname{sign}\left(\dot{\theta}_{m}\right)+f_{v} \dot{\theta}_{m},
$$

with $f_{c}$ and $f_{v}$ being the Coulomb and viscous coefficient, respectively.

\section{A. Friction observer}

The structure of the friction observer (Fig. 3) is inspired by the momentum-based collision detection algorithms developed in [9], [10], where it is used for detecting external disturbance torques acting on the rigid robot dynamics. Here the algorithm is adapted to the much simpler, linear actuator dynamics, for which the friction torque constitutes the disturbance. The observer dynamics is given by

$$
\begin{aligned}
u_{m} & =J_{m} \ddot{\hat{\theta}}_{m}+\tau_{a m}+\hat{\tau}_{f m} \\
\hat{\tau}_{f m} & =-L J_{m}\left(\dot{\theta}_{m}-\dot{\hat{\theta}}_{m}\right),
\end{aligned}
$$

\footnotetext{
${ }^{1}$ The robot dynamics includes the rigid body dynamics and elastic transmission elements related to the gear-box and/or the torque sensor.

${ }^{2}$ See also Fig. 6.
}

with $L>0$, and where $\hat{\tau}_{f m}$ and $\dot{\hat{\theta}}_{m}$ are the estimation of the friction and the observer state, respectively. By combining (1) with (5) and (6), one obtains

$$
\hat{\tau}_{f m}=\frac{1}{L^{-1} s+1} \tau_{f m},
$$

where $s$ is the Laplace operator. The estimated friction corresponds thus to the actual friction passed through a first order filter. The friction compensation is obtained by adding the estimated friction to the control torque.

$$
u_{m}=u_{c m}+\hat{\tau}_{f m},
$$

with $u_{c m}$ being the torque generated by the controller.

We note that the observer has a very simple structure due to the measurement of both motor position (with numerically differentiated velocity) and elastic joint torque. Therefore the information about the nonlinear rigid body model is not required for the observer.

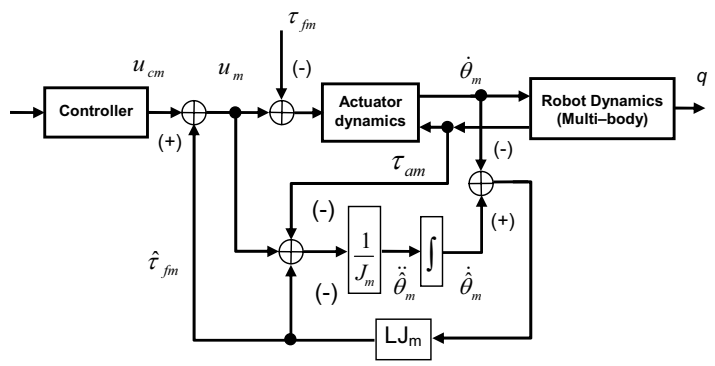

Fig. 3. Friction observer and compensation structure for the multi-dof case.

\section{B. Passivity analysis}

Assume that one has a controller, which provides asymptotic stability for the system without friction. The question is whether the above introduced friction compensation ensures the stability and the convergence of the controlled system with friction. For linear systems, the stability of various versions of disturbance observers has been well studied [11], [12], [13] and it can be easily shown that the observer corresponds to an integral action driving the steady state error to zero. For example, a PD controller combined with this friction observer would lead to a PID-like controller. However, in our case we will consider the actuator as part of a more complex MIMO controller in a nonlinear robotic system, as described in the next section. Therefore, we cannot apply linear methods and we will instead analyze the passivity properties of the friction compensation. If it turns out to be passive, the observer can be easily incorporated into a Lyapunov stability analysis of the robot with a passive controller.

With the friction compensation (8), eq. (3) becomes

$$
\dot{S}_{\theta}=\dot{\theta}_{m} u_{c m}-\dot{\theta}_{m} \tau_{a m}+\dot{\theta}_{m}\left(\hat{\tau}_{f m}-\tau_{f m}\right)
$$

and we want to check if $\dot{\theta}_{m}\left(\hat{\tau}_{f m}-\tau_{f m}\right)$ is still negative, i.e., is dissipating energy, while being able to avoid steady state errors usually caused by Coulomb friction.

Due to the linearity of the filtering operation, the friction estimation will contain a component corresponding to the 
Coulomb friction and one corresponding to the viscous friction

$$
\hat{\tau}_{f m}=\hat{\tau}_{f m, c}+\hat{\tau}_{f m, v}
$$

with

$$
\begin{aligned}
\hat{\tau}_{f m, c} & =\frac{1}{L^{-1} s+1} \tau_{f m, c} \\
\hat{\tau}_{f m, v} & =\frac{1}{L^{-1} s+1} \tau_{f m, v} .
\end{aligned}
$$

In the following, the notation

$$
\hat{\tau}_{f m, v} \triangleq f_{v} \nu_{m}
$$

will be used, where

$$
\nu_{m}=\frac{s}{L^{-1} s+1} \theta_{m}
$$

is the filtered motor velocity.

As can be easily recognized also in Fig. 4, for the Coulomb friction compensation one has the property

$$
\dot{\theta}_{m}\left(\hat{\tau}_{f m, c}-\tau_{f m, c}\right) \leq 0 .
$$

Indeed, the absolute value of $\hat{\tau}_{f m, c}$ is always smaller than the absolute value ${ }^{3}$ of $\tau_{f m, c}$ and the difference always has the opposed sign of $\dot{\theta}_{m}$. Therefore, this term is always dissipative.

For the passivity analysis of the case including also viscous friction compensation, one has to add to the storage function (2) the energy corresponding to the filter state, leading to

$$
S_{1 \theta}=\frac{1}{2} J_{m} \dot{\theta}_{m}^{2}+\frac{1}{2} f_{v} L^{-1} \nu_{m}^{2}
$$

The derivative of this storage function for the considered friction model becomes

$$
\dot{S}_{1 \theta}=\dot{\theta}_{m} u_{c m}-\dot{\theta}_{m} \tau_{a m}+P_{\text {fric }}
$$

where the power $P_{\text {fric }}$ dissipated by the friction and the friction compensation is obtained using (14)

$$
P_{\text {fric }}=-\dot{\theta}_{m}\left(\tau_{f m, c}-\hat{\tau}_{f m, c}\right)-f_{v} \dot{\theta}_{m}^{2}-f_{v} \nu_{m}^{2} \leq 0,
$$

which is negative, as desired.

${ }^{3}$ Note that the Coulomb friction represents a step input signal to the first order filter.
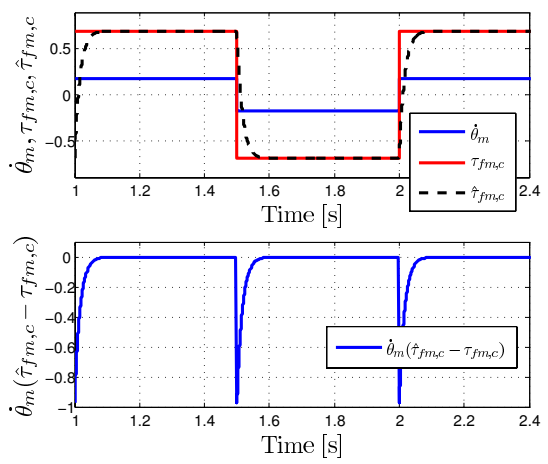

Fig. 4. Coulomb friction compensation still ensures energy dissipation.

\section{Limitation of the approach}

While the friction observer will always provide a filtered friction signal, the friction compensation will not be passive for any friction profile. This can be seen in Fig. 5 for the case of stiction (Stribeck effect). The filtered friction becomes temporarily higher than the real friction, leading therefore to an overcompensation of friction and thus to energy generation. This might result in limit cycles for the system. A possible solution is to monitor the energy corresponding to friction and friction compensation over a period of time $t-t_{0}$

$$
E_{\text {fric }}(t)=S_{1 \theta}(t)-S_{1 \theta}\left(t_{0}\right)-\int_{t_{0}}^{t} \dot{\theta}_{m}\left(u_{c m}-\tau_{a m}\right) \mathrm{d} t
$$

and to switch off or scale down the friction compensation if the energy exceeds a certain positive threshold.

\section{Modelling of the Medical Robot}

The DLR medical robot in Fig. 1 has $n=7$ rotary joints that exhibit considerable elasticity. Apart from the first joint, the following three joint pairs are coupled with differential gears. The following dynamic model was introduced in [14]

$$
\begin{aligned}
u_{m} & =J_{m} \ddot{\theta}_{m}+T^{T} \tau_{a}+\tau_{f m} \\
\tau_{a} & =M(q) \ddot{q}+C(q, \dot{q}) \dot{q}+g(q)
\end{aligned}
$$

with

$$
\begin{aligned}
\tau_{a} & =\tau+D K^{-1} \dot{\tau} \\
\tau & =K\left(T \theta_{m}-q\right) .
\end{aligned}
$$

Therein, $q \in R^{n}$ and $\theta_{m} \in R^{n}$ are the link and motor angles, respectively. The elastic torque vector $\tau \in R^{n}$ is determined by a the linear relationship $\tau=K\left(T \theta_{m}-q\right)$ and is measured by strain gauge based torque sensors, while the total joint torque vector is denoted by $\tau_{a}$. The joint stiffness matrix $K \in R^{n \times n}$ and the joint damping matrix $D \in R^{n \times n}$ are
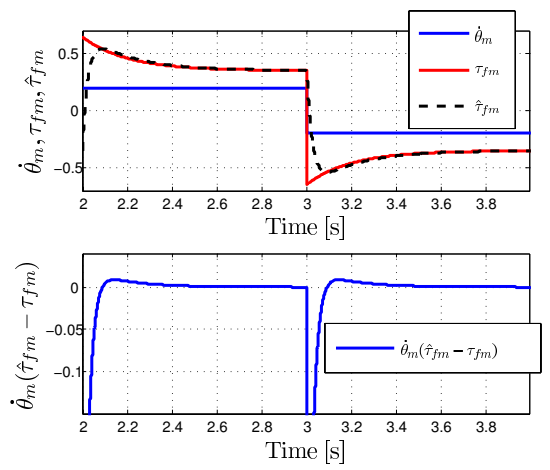

Fig. 5. Stiction compensation does not ensure energy dissipation. 
symmetric and positive definite and have following structure:

$$
\begin{aligned}
& \mathcal{P}=\left[\begin{array}{cccc}
\mathcal{P}_{1} & \ldots & & 0 \\
& \mathcal{P}_{2} & & \\
\vdots & & \mathcal{P}_{4} & \vdots \\
0 & \ldots & & \mathcal{P}_{6}
\end{array}\right] \\
& \mathcal{P}_{1} \in R, \mathcal{P}_{i} \in R^{2 \times 2}, \\
& \text { with } i=\{2,4,6\}, \mathcal{P}=\{K, D\} .
\end{aligned}
$$

The motor inertia matrix $J_{m} \in R^{n \times n}$ is diagonal as well. $M(q) \in R^{n \times n}$ is the mass matrix, $C(q, \dot{q}) \in R^{n \times n}$ the centrifugal and Coriolis vector, and $g(q) \in R^{n}$ the gravity vector of the rigid body model. The control input is the motor torque $u_{m} \in R^{n}$. $\tau_{f m} \in R^{n}$ is the friction torque [3], [14]. Due to the coupling of the joints through the differential gear, one has to distinguish between motor coordinates, denoted by the subscript $m$, and coordinates after the gearbox (or, on the link side), written without subscripts. For example, the motor position as well as the motor inertia are given in motor coordinates, while the joint torque is measured after the gear, in link coordinates. Due to the differential gears, the transformations between motor and link coordinates for positions and torques are given by

$$
\left\{\begin{array}{c}
\theta=T \theta_{m} \\
\tau_{a m}=T^{T} \tau_{a}
\end{array}\right.
$$

with

$$
T=\left[\begin{array}{cccc}
1 & \ldots & & 0 \\
& \mathcal{T} & & \\
\vdots & & \mathcal{T} & \vdots \\
0 & \ldots & & \mathcal{T}
\end{array}\right] \quad, \forall \mathcal{T}=\left[\begin{array}{cc}
\frac{1}{2} & \frac{1}{2} \\
-\frac{1}{2} & \frac{1}{2}
\end{array}\right]
$$

Finally, for the convergence analysis, the following standard property of the gravity vector is used:

$$
\exists \alpha>0 \mid\left\|g\left(q_{1}\right)-g\left(q_{2}\right)\right\| \leq \alpha\left\|q_{1}-q_{2}\right\| \quad \forall q_{1}, q_{2} .
$$

To better highlight the relation between the robot model and

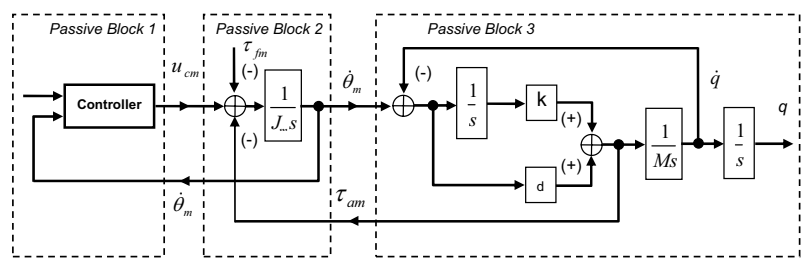

Fig. 6. Passive representation of a linear, 1 dof flexible joint.

the 1 dof analysis from Sec. II and in particular to Fig. 2, the passive representation of a single linear flexible joint is given in Fig. 6.

\section{Convergence AnAlysis of THE MiMO}

\section{CONTROLLER WITH EXACT FRICTION COMPENSATION}

The controller structure and the asymptotic stability results obtained in [14] will be summarized here for the case of exact friction compensation.
For the controller design, the actuator dynamics (20) is rewritten in terms of link coordinates (after the gearbox) as

$$
u=J \ddot{\theta}+\tau_{a}+\tau_{f} .
$$

The following tensor transformations have been used

$$
\left\{\begin{array}{l}
u=T^{-T} u_{m} \\
\theta=T \theta_{m} \\
J=T^{-T} J_{m} T^{-1} \\
\tau_{f}=T^{-T} \tau_{f m}
\end{array}\right.
$$

Notice that $J \in R^{n \times n}$, the motor inertia matrix written in link coordinates, is positive definite and symmetric, but in general not diagonal. The transformed robot dynamics is

$$
\begin{aligned}
u & =J \ddot{\theta}+\tau_{a}+\tau_{f} \\
\tau_{a} & =M(q) \ddot{q}+C(q, \dot{q}) \dot{q}+g(q)
\end{aligned}
$$

with

$$
\begin{aligned}
\tau_{a} & =\tau+D K^{-1} \dot{\tau} \\
\tau & =K(\theta-q) .
\end{aligned}
$$

In these equations, the stiffness and damping matrices $K$ and $D$ of the coupled joints are not diagonal, due to the coupling of the joints, as described in [14]. The following linear state feedback controller ${ }^{4}$ is used for the medical robot

$$
u=u_{c}+\tau_{f}
$$

with

$$
\begin{aligned}
u_{c}= & K_{P} \widetilde{\theta}-K_{D} \dot{\theta}-K_{T} K^{-1} \tau-K_{S} K^{-1} \dot{\tau} \\
& +\left(K+K_{T}\right) K^{-1} g\left(q_{d}\right),
\end{aligned}
$$

where $\tilde{\theta}=\theta_{d}-\theta$. The matrices $K_{C}$, with $C \in\{P, D, T, S\}$, are diagonal and positive definite and have the same structure as in (24).

By substituting (34-35) into (30) one obtains the dynamics of the closed loop system containing the actuator and the controller:

$$
\begin{aligned}
J \ddot{\theta}= & K_{P} \tilde{\theta}-K_{D} \dot{\theta}-\left(K_{T}+K\right) K^{-1} \tau \\
& -\left(K_{S}+D\right) K^{-1} \dot{\tau}+\left(K+K_{T}\right) K^{-1} g\left(q_{d}\right)
\end{aligned}
$$

The following Lyapunov function was used for stability analysis

$$
\begin{aligned}
V_{1}(\theta, \dot{\theta}, q, \dot{q})= & \frac{1}{2} \dot{\theta}^{T} K\left(K+K_{T}\right)^{-1} J \dot{\theta} \\
& +\frac{1}{2} \dot{q}^{T} M(q) \dot{q}+\frac{1}{2}(\widetilde{\theta}-\widetilde{q})^{T} K(\widetilde{\theta}-\widetilde{q}) \\
& +\frac{1}{2} \widetilde{\theta}^{T} K\left(K+K_{T}\right)^{-1} K_{P} \widetilde{\theta} \\
& +U_{g}(q)-U_{g}\left(q_{q}\right)+\widetilde{q}^{T} g\left(q_{d}\right)
\end{aligned}
$$

with $\widetilde{q}=q_{d}-q . U_{g}(q)$ is the potential energy of the gravity field. This Lyapunov function contains the kinetic energy of the motors and links, and the potential energy of the joint springs, of the gravity field, and of the controller

\footnotetext{
${ }^{4}$ Due to the fourth-order dynamics of flexible joint robots, a complete state is given by the motor position $\theta$ and velocity $\dot{\theta}$, as well as by the torque $\tau$ and its derivative $\dot{\tau}$.
} 
springs. Moreover, note that the kinetic energy contains the motor inertia scaled down by the torque feedback gain. This corresponds to the interpretation of the torque feedback as a shaping of the motor inertia [15].

In [14] it has been shown that the Lyapunov function is positive definite if the following condition is fulfilled

$$
\alpha I<K\left(K+K_{T}+K_{P}\right)^{-1} K_{P} .
$$

Loosely speaking, this condition requires that the controlled robot can sustain itself in the gravity field.

The derivative of the Lyapunov function along the system trajectories is:

$$
\begin{aligned}
\dot{V}_{1}= & -\dot{\theta}^{T} K\left(K+K_{T}\right)^{-1}\left(K_{D}+K_{S}+D\right) \dot{\theta}-\dot{q}^{T} D \dot{q} \\
& +\dot{q} D \dot{\theta}+\dot{\theta}^{T} K\left(K+K_{T}\right)^{-1}\left(K_{S}+D\right) \dot{q}
\end{aligned}
$$

In [14] it was shown that $\dot{V}_{1}$ is negative definite for large enough $K_{D}$. Using LaSalle invariance principle, it can be shown that the robot converges to the maximal invariant set $\left[\theta=\theta_{d}, \dot{\theta}=0, q=q_{d}, \dot{q}=0\right]$. This implies global asymptotic stability.

\section{Stability of The MiMO controlled System WITH FRICTION OBSERVER}

Based on the passivity of the friction compensation, it is straightforward to show the stability of any system containing a passive plant, a passive controller and the friction compensation, and for which asymptotic stability can be shown in absence of friction (or, equivalently, assuming exact friction compensation). The interesting point with the presented state feedback controller is that while the position and velocity feedback terms have a simple passivity based interpretation (as spring and damper), the torque feedback itself does not represent a passive controller component. However, as shown e.g. in [15], the torque feedback can be interpreted as scaling of the actuator dynamics. This can be also easily recognized when multiplying (36) by $K\left(K+K_{T}\right)^{-1}$ or by looking at the Lyapunov function (37), in which the motor inertia and the potential energy of the controller spring are scaled down by the same factor. When considering the friction together with the friction compensation from (8), the closed-loop actuator dynamics becomes

$$
\begin{aligned}
J \ddot{\theta}= & K_{P} \widetilde{\theta}-K_{D} \dot{\theta}-\left(K+K_{T}\right) K^{-1} \tau \\
& -\left(K_{S}+D\right) K^{-1} \dot{\tau}+\left(K+K_{T}\right) K^{-1} g\left(q_{d}\right) \\
& +\hat{\tau}_{f}-\tau_{f} .
\end{aligned}
$$

If one analyzes a Lyapunov function $V_{2}=V_{1}$, with the new actuator dynamics (40), one has

$$
\dot{V}_{2}=\dot{V}_{1}+\dot{V}_{12}
$$

with

$$
\dot{V}_{12}=\dot{\theta}^{T} K\left(K+K_{T}\right)^{-1}\left(\hat{\tau}_{f}-\tau_{f}\right)
$$

Property (15) is valid only at the level of each actuator at which the friction is really acting, but not in the link coordinates. Therefore one needs first to express (42) in motor coordinates, as

$$
\begin{aligned}
\dot{V}_{12} & =\dot{\theta}_{m}^{T} A\left(\hat{\tau}_{f m}-\tau_{f m}\right) \\
& =\dot{\theta}_{m}^{T} A\left[\left(\hat{\tau}_{f m, c}-\tau_{f m, c}\right)+f_{v} \nu_{m}-f_{v} \dot{\theta}_{m}\right]
\end{aligned}
$$

with

$$
A=T^{T} K\left(K+K_{T}\right)^{-1} T^{-T} .
$$

In order to apply (15), the condition has to be found, for which $A \in R^{n \times n}$ is diagonal and positive definite. Obviously this is the case if

$$
K_{T}=\beta K, \quad \beta>0 .
$$

One can also show (by imposing that the $2 \times 2$ coupled submatrices of $A$ have zero off-diagonal terms and solving the resulting equations) that (45) is also a necessary condition. Therefore one can conclude that, for preserving passivity, $K_{T}$ might differ from $K$ only by a positive factor. Now A has the form $A=(\beta+1) I$. Similarly to Sec. II.B, consider the Lyapunov function including the filtered energy

$$
V=V_{1}+\frac{1}{2} \nu_{m}^{T} L^{-1} A f_{v} \nu_{m}
$$

Its derivative is

$$
\dot{V}=\dot{V}_{1}+\sum_{i} a_{i} P_{\text {fric } i} \leq 0
$$

with $a_{i}$ being the diagonal elements of $A$. Therefore, according to Sec. II, $\dot{V} \leq 0$ and the system is stable.

In order to analyze the convergence of the system one has to find the equilibrium points obtained from the system equations for $\left[\dot{\theta}=0, \dot{q}=0, \nu_{m}=0\right]^{T}$. The equilibrium equations are

$$
\begin{array}{r}
K_{P} \tilde{\theta}-\left(K+K_{T}\right)(\theta-q)+\left(K+K_{T}\right) K^{-1} g\left(q_{d}\right) \\
+T^{-T}\left(\hat{\tau}_{f m, c}-\tau_{f m, c}\right)=0 \\
K(\theta-q)=g(q)
\end{array}
$$

Note that at steady state $\hat{\tau}_{f m, c}-\tau_{f m, c}=0$ holds and therefore the equilibrium equations are the same as for exact friction compensation. This is not surprising, since the friction compensation provides exact friction compensation at steady state. According to LaSalle invariance principle, the system converges to the largest invariant set, which is given by the unique point $\left[\theta=\theta_{d}, \dot{\theta}=0, q=q_{d}, \dot{q}=0, \nu_{m}=\right.$ $\left.0, \tau_{f m, c}=\hat{\tau}_{f m, c}\right]$. The system is therefore global asymptotically stable under the same conditions as in Sec. IV.

Remark: The main lesson learned from this analysis is that there is a substantial qualitative difference between a controller which is passive by itself (like our PD term), and a controller which is itself not passive, but can be shown to provide a passive subsystem together with (part of) the plant dynamics, as for the torque feedback in our case. The first will lead to stability for any passive plant, also for passive, but un-modelled dynamics, e.g. friction. This very convenient robustness property of all passive controllers gets largely lost for passivity-based controllers. We have seen that a torque feedback with general, non-diagonal $K_{T}$ is not passive any more with respect to friction. The same situation is often encountered in literature, e.g. for passivity based tracking controllers [16]. Maybe a clear terminological distinction has to be done between passive controllers and passivitybased controllers. It needs to be carefully analyzed to which extent the robustness property really applies to a certain passivity-based controller. 


\section{EXPERIMENTS}

In this section two experimental results for the MIMO controller with and without friction compensation are presented. Some of the relevant parameters of the robot used in the experiments, including the coefficients needed in modelbased friction compensation, are listed in Table I. In the first experiment, the robot follows a periodic trajectory (see Fig. 7) in order to show the behavior in terms of tracking errors. Figure 8 and 9 show, respectively, the friction torques and the position errors for a controller without friction compensation, with a model-based friction compensation, and with a compensation using the proposed friction observer. The best performance is uniformly obtained by the controller using the friction observer. While the errors at joints 1, 4, 5, 6 , and 7 are all below $0.01 \mathrm{deg}$, the errors at joints 2 and 3 are slightly worse due to a small backlash in this prototype. For the point to point motion task shown in Fig. 10, the model based compensation cannot be used due to limit cycles around the end point. The cases with and without friction observer are shown in Fig. 11 and Fig. 12. For the joints 1 and 4 to 7 , the position error with friction observer is again much lower. For joints 2 and 3, errors between the measured and model torque still cause some steady state error.

It can be concluded that the friction observer considerably contributes to the reduction of the positioning errors.

\section{CONCLUSIONS}

We proposed in this paper a friction observer and friction compensation method that can be used together with passivity-based controllers in order to enhance the robot accuracy. The friction compensation, though similar to an integral action from the point of view of performance in free motion, has several advantages. First, it avoids saturation or overflow of the integrator in case of external disturbance torques (e.g. unexpected contacts). Second, only friction is compensated, instead of the sum of friction and external disturbance, so that it can be used also by impedance control in contact with the environment. Third, the design of the friction observer can be done independently of the MIMO controller design, whereas when adding an integrator all gains of the controllers have to be changed for good performance. Finally, our approach preserves the global asymptotic stability of the original MIMO controller even in the presence of friction. Experimental results validate the approach for the DLR medical robot.

\section{ACKLOWLEDGEMENT}

This work has been partially funded by the European Commissions Sixth Framework Programme as part of the

TABLE I

ROBOT AND CONTROL PARAMETERS USED IN THE EXPERIMENTS

\begin{tabular}{|l|c|c|c|c|}
\hline & $J\left(\mathrm{kgm}^{2}\right)$ & $f_{c}(\mathrm{Nm})$ & $f_{v}\left(\mathrm{~N} / \mathrm{s}^{2}\right)$ & $L$ \\
\hline joint 1 & 0.6504 & 1.503 & 5.640 & 700 \\
joint 2 & 1.2681 & 6.731 & 24.22 & 400 \\
joint 3 & 1.2681 & 9.332 & 27.15 & 400 \\
joint 4 & 0.2984 & 4.446 & 3.820 & 700 \\
joint 5 & 0.2984 & 3.339 & 3.160 & 700 \\
joint 6 & 0.2984 & 4.103 & 1.160 & 700 \\
joint 7 & 0.2984 & 4.106 & 1.770 & 700 \\
\hline
\end{tabular}

PHRIENDS project under grant no. 045359.

\section{REFERENCES}

[1] T. Ortmaier, H. Wess, U. Hagn, M. Grebenstein, M. Nickl, A. AlbuSchäffer, C. Ott, S. Jörg, R. Konietschke, L. Le-Tien and G. Hirzinger, "A Hands-On-Robot for Accurate Placement of Pedicle Screws." IEEE Int. Conf. on Robotics and Automation, 4179-4186, 2006.

[2] B. S. R. Armstrong, "Dynamics for Robot Control: Friction Modelling and Ensuring Excitation During Parameter Identification." Dissertation, Stanford University, 1988.

[3] A. Albu-Schäffer, "Regelung von Robotern mit elastischen Gelenken am Beispiel der DLR-Leichtbauarme." In Dissertation, Technische Üniversität München, April, 2002.

[4] Q. H. Xia, S. Y. Lim, M. H. A. Jr, T. M. Lim, "Adaptive Joint Friction Compensation Using a Model-Based Operational Space Velocity Observer." IEEE Int. Conf. on Robotics and Automation, pp. 3081-3086, 2004

[5] P. Tomei, "Robust Adaptive Friction Compensation for Tracking Control of Robot Manipulators." IEEE Trans. on Automatic Control, vol. 45, pp. 2164-2169, 2000.

[6] V. Parra-Vega, S. Arimoto, "A passivity based adaptive sliding mode position-force control for robot manipulators." Int. J. of Adaptive Control and Signal Processing, pp. 365-377, 1996.

[7] C. C. de Wit, H. Olsson, K. J. Astron, P. Linschinsky, "A New Model for Control of Systems with Friction." IEEE Trans. on Automatic Control, vol. 40, pp. 419-425, 1994.

[8] S. Arimoto, "State-Of-The-Art and Future Research Direction of Robot Control." IFAC Symposium on Robot Control, pp. 3-14, 1994.

[9] A. De Luca, R. Mattone, "Actuator Failure Detection and Isolation Using Generalized Momenta." IEEE Int. Conf. on Robotics and Automation, pp. 634-639, 2003.

[10] A. De Luca, A. Albu-Schäffer, S. Haddadin, G. Hirzinger, "Collision Detection and Safe Reaction with the DLR-III Lightweight Manipulator Arm." IEEE/RSJ Int. Conf. on Intelligent Robots and Systems, pp. 16231630, 2006.

[11] J. R. Ryoo, T-Y. Doh, M. J. Chung, ”Disturbance Observer Design for Track-following Control in Optical Disk Drive using Structured Singular Value." Int. Conf. on Control, Automation and Systems, Korea, pp. 1407-1412, 2002.

[12] K. Yang, Y. Choi, W. K. Chung, I. H. Suh, S. R. Oh, "Robust Tracking Control of Optical Disk Drive Systems Using Error based Disturbance Observer and its Performance Measure." Proceedings of the American Control Conference, Anchorage, pp. 1395-1400, 2002.

[13] N. Bajcinca, R. Cortesao, M. Hauschild, "Haptic Control for Steer-byWire Systems." IEEE/RSJ Int. Conf. on Intelligent Robots and Systems, pp. 2004 - 2009, 2003.

[14] L. Le-Tien, A. Albu-Schäffer, G. Hirzinger, "MIMO State Feedback Controller for a Flexible Joint Robot with Strong Joint Coupling." IEEE Int. Conf. on Robotics and Automation, pp. 3824-3830, 2007.

[15] A. Albu-Schäffer, C. Ott, G. Hirzinger, "A unified passivity-based control framework for position, torque and impedance control of flexible joint robots." The Int. J. of Robotics Research, Vol. 26, Nr. 1, pp. 23-39, 2007.

[16] B. Brogliato, R. Ortega und R. Lozano, "Global Tracking Controllers for Flexible-Joint Manipulators: a Comparative Study." Automatica, vol. 31, pp. 941-956, 1995.

[17] Y. Wang, Z. Xiong, H. Ding, X. Zhu, "Nonlinear Friction Compensation and Disturbance Observer for a High-speed Motion Platform." IEEE Int. Conf. on Robotics and Automation, pp. 4515-4520, 2004.

[18] C. Makkar, W. E. Dixon, W. G. Sawyer, G. Hu, "Lyapunov-Based Tracking Control in the Presence of Uncertain Nonlinear Parameterizable Friction." American Control Conference, pp. 1975-1980, 2005.

[19] J-J. E. Slotine, W. Li, “Applied Nonlinear Control.” Prentice-Hall International, Inc., 1991.

[20] A. R. G. Ramirez, A. L. D. Franco, E. R. De Pieri, R. Guenther, "Friction Modelling, Identification and Compensation." Mechatronics, 2007.

[21] P. Tomei, "Robust Adaptive Control for Robots with Arbitrary Transient Performance and Disturbance Attenuation." IEEE Trans. on Automatic Control, vol. 44, pp. 654-658, 1999. 


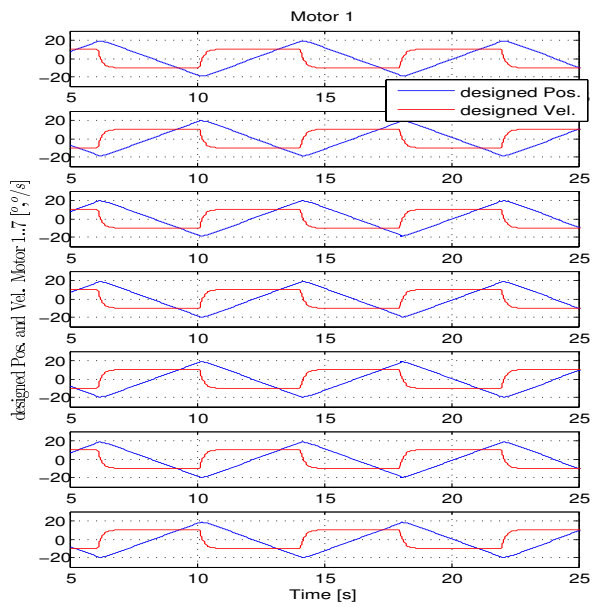

Fig. 7. Periodic trajectory: Position and velocity profiles.

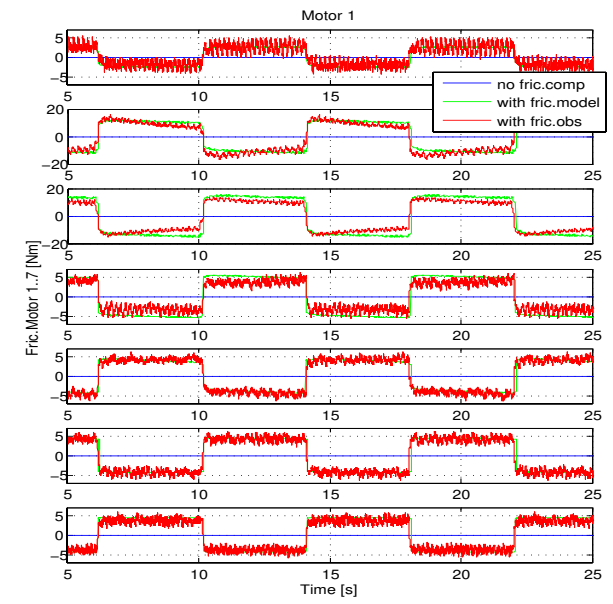

Fig. 8. Friction compensation torques from model and from observer.

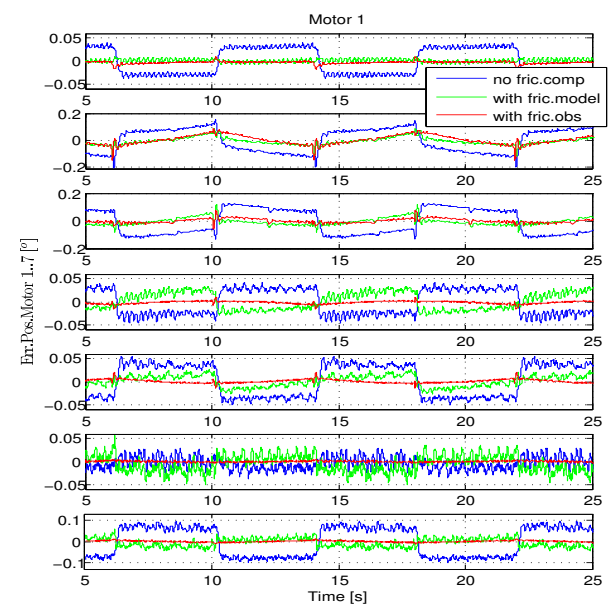

Fig. 9. Tracking errors for periodic trajectory.

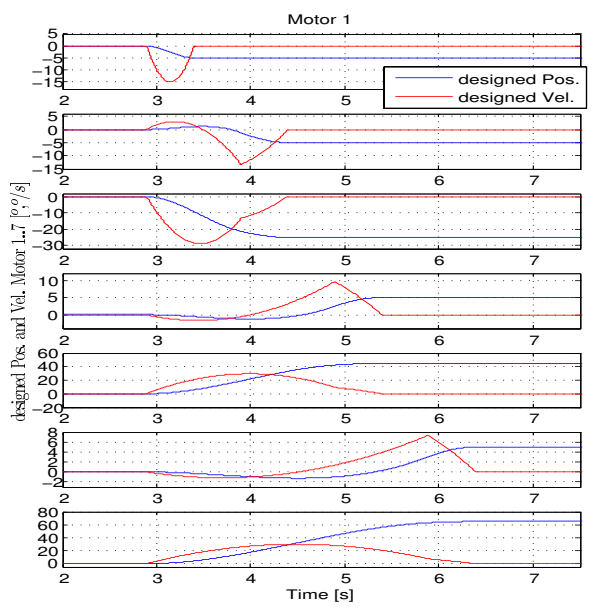

Fig. 10. Point to point trajectory: Position and velocity profiles.

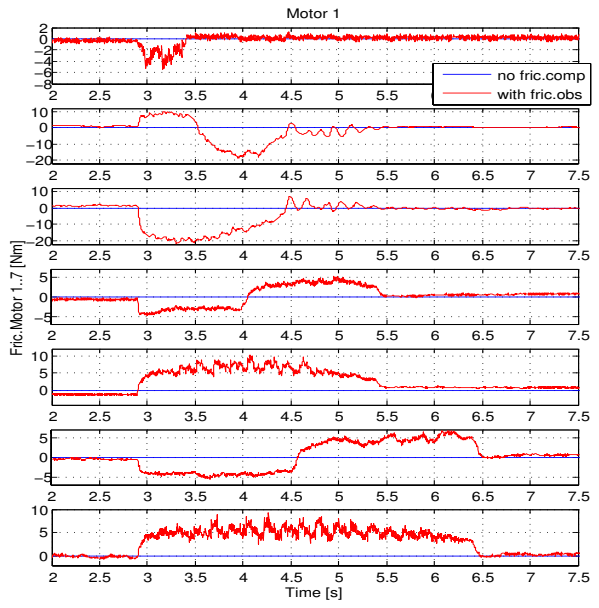

Fig. 11. Friction compensation torques from observer

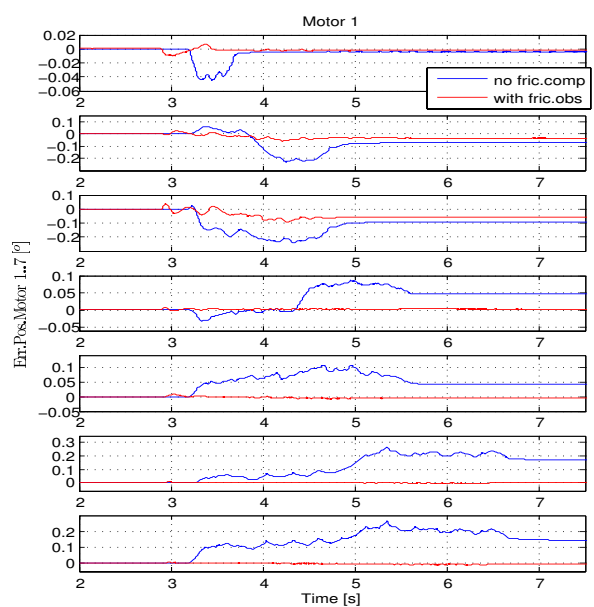

Fig. 12. Position errors for point to point trajectory. 